Open Access

\title{
Digital imaging in the immunohistochemical evaluation of the proliferation markers Ki67, MCM2 and Geminin, in early breast cancer, and their putative prognostic value
}

Shalaka Joshi ${ }^{1,2,4^{*}+}$, Johnathan Watkins ${ }^{1,2+}$, Patrycja Gazinska ${ }^{1,2}$, John P. Brown ${ }^{1}$, Cheryl E. Gillett ${ }^{1,3}$, Anita Grigoriadis ${ }^{1,2}$ and Sarah E. Pinder ${ }^{1,3}$

\begin{abstract}
Background: Immunohistochemical assessment of proliferation may provide additional prognostic information in early breast cancer. However, due to a lack of methodological standards proliferation markers are still not routinely used for determining therapy. Even for Ki67, one of the most widely-studied markers, disagreements over the optimal cutoff exist. Improvements in digital microscopy may provide new avenues to standardise and make data more reproducible.

Methods: We studied the immunohistochemical expression of three markers of proliferation: Ki67, Mini-Chromosome Maintenance protein 2 and Geminin, by conventional light microscope and digital imaging on triplicate TMAs from 309 consecutive cases of primary breast cancers. Differences between the average and the maximum percentage reactivity in tumour cell nuclei from the three TMA cores were investigated to assess the validity of the approach. Time-dependent Receiver Operating Characteristic curves were utilized to obtain optimal expression level cut-offs, which were then correlated with clinico-pathological features and survival.

Results: High concordance between conventional and digital scores was observed for all 3 markers (Ki67: $r_{s}=0.87, P<0.001 ; M C M 2: r_{s}=0.94, P<0.001$; and Geminin: $r_{s}=0.86, P<0.001$; Spearman's rank). There was no significant difference according to the number of TMA cores included for either Ki67 or MCM2; analysis of two or three cores produced comparable results. Higher levels of all three proliferation markers were significantly associated with higher grade $(P<0.001)$ and ER-negativity $(P<0.001)$. Optimal prognostic cut-offs for percentage expression in the tumour were $8 \%, 12$ and $2.33 \%$ for Ki67, MCM2 and Geminin respectively. All 3 proliferation marker cutoffs were predictive of 15-year breast cancer-specific survival in univariable Cox regression analyses. In multivariable analysis only lymph node status ( $\mathrm{HR}=3.9,95 \% \mathrm{Cl}=1.79-8.5, P=0.0006)$ and histological grade $(\mathrm{HR}=1.84,95 \% \mathrm{Cl}=1-3.38, P=0.05)$ remained significantly prognostic.

(Continued on next page)
\end{abstract}

\footnotetext{
* Correspondence: drjoshishalaka@gmail.com

${ }^{\dagger}$ Equal contributors

'Department of Research Oncology, King's College London, Faculty of Life Science and Medicine, Division of Cancer Studies, Bermondsey Wing, Guy's Hospital, London, UK

${ }^{2}$ Breast Cancer Now Unit, King's College London, Faculty of Life Science and Medicine, Division of Cancer Studies, Bermondsey Wing, Guy's Hospital, London, UK

Full list of author information is available at the end of the article
} 
(Continued from previous page)

Conclusions: Here we show that. MCM2 is a more sensitive marker of proliferation than Ki67 and should be examined in future studies, especially in the lymph node-negative, hormone receptor-positive subgroup. Further, digital microscopy can be used effectively as a high-throughput method to evaluate immunohistochemical expression.

Keywords: Ki67, MCM2, Geminin, Proliferation, Digital microscopy, Immunohistochemistry, Survival analysis

\section{Background}

Breast cancer is a heterogeneous disease [1]. With earlier detection and improved treatment options, breast cancer-related mortality is decreasing, while the detection of early stage disease is on the rise [2]. Traditional prognostic and predictive factors such as lymph node status, histological grade, invasive tumour size, hormone receptor (ER and PR) and HER2 status may be insufficient for prognosticating early stage disease [3, 4]. As such, there is a need for better markers to categorise primary, operable breast cancers and reduce overtreatment in those patients with a good prognosis, and offer more aggressive treatment regimes to those in the poor prognosis group.

Proliferation is one of the most fundamental properties of cancer [5]. Histological grade is an important prognostic marker, which reflects proliferation status by incorporating an assessment of mitotic rate. Other methods of assessing proliferation, such as S-phase fraction, mitotic activity index (MAI) and radionucleotide labeling indices have limitations, and have not proven to be of utility over and above the prognostic value of histological grade, and consequently, they have not been applied in clinical practice [6]. Ki67 has been one of the most extensively studied proliferation markers since its discovery in the early 1980s [7]. Since the development of the MIB-1 antibody, immunohistochemical expression of $\mathrm{Ki} 67$ in paraffin-embedded tissue has been shown in a number of studies to be prognostic and predictive of treatment response in breast cancer [8-10].

Molecular profiling of breast cancer can be used to classify early breast cancer into prognostic groups [1]. Ki67 measured by immunohistochemistry (IHC) has been proposed to be a useful surrogate for molecular subtype. Ki67 at a cut-off of $13.25 \%$ can identify and divide ER-positive breast cancers into the luminal A and B subgroups with moderate accuracy and with a significant difference in patient survival [11]. As a result, the St Gallen guidelines recommend a cut-off of $14 \%$ for Ki67 in deciding how to manage early breast cancer patients in the adjuvant treatment setting [12]. Other studies have reported that immunohistochemical analysis of ER, PR, HER-2 and Ki67 (the latter at a cut-off of $10 \%$ ), and a derived IHC-4 score is equivalent to the 21-gene recurrence score that is the basis of Oncotype-DX in predicting recurrence and survival in ER-positive breast cancer $[13,14]$. Currently, trials are underway to stratify hormone receptor-positive, early breast cancer patients by their gene expression profile into those with a low or high risk of recurrence [15], which in turn influences the decision to administer chemotherapy. Of note, 5 of the 21 genes assessed in Oncotype-DX are proliferation genes, emphasising the importance of proliferation status in tumour prognostication and in clinical decision making [16]. Gwin et al. studied the correlation of Ki67 expression assessed by IHC in 32 breast cancer patients for possible association with the Oncotype-DX's recurrence score (RS) and found it to be high in some of the low RS cases, as a result of which they suggested that Ki67 be used alongside the RS [17].

Other markers of proliferation have been identified as participants in the process of DNA replication as well as exhibiting prognostic value. Mini-chromosome maintenance (MCM) proteins are DNA helicases that, along with the Origin Recognition Complex (ORC) and Cdc6p, form the pre-Replication Complex (pre-RC), to initiate DNA replication [18]. The dissociation of MCM proteins from the pre-RC is controlled by Geminin, which prevents rereplication by inhibiting Cdt-1 [19]. The immunohistochemical expression of these proteins has been correlated with prognosis in breast and other cancers [20-22].

However, methodological variability in assessing these proliferation markers represents one of the main difficulties for translating these research findings into the clinic. Consequently, in an attempt to standardise the technique, the "International Ki67 in Breast Cancer Working Group" has drafted guidelines for the immunohistochemical assessment of Ki67 [23]. Adhering to these criteria, we carried out a study to evaluate two different methods of assessing Ki67, MCM2 and Geminin IHC in tissue microarrays (TMAs) of a series of consecutive invasive breast cancer cases. We aimed to evaluate the concordance between conventional microscopic methods (i.e. the histological sections) and digital scanned images from the same material applied to three markers of proliferation. Having evaluated the similarity between the 
two scoring methodologies, we sought to compare the expression patterns of the three proliferation markers with each other in order to establish their ability to capture tumour proliferation status, as well as to determine their association with clinico-pathological characteristics.

\section{Methods}

\section{Patients}

Formalin-fixed paraffin-embedded (FFPE) tissue blocks were retrieved from 309 patients who presented with primary invasive breast cancer between December 1989 and September 1992 to Guy's and St Thomas' Breast Unit. Unless there was insufficient tissue for research purposes, consecutive cases were selected, All patients were treated surgically, either in the form of modified radical mastectomy or breast conservation surgery, followed by adjuvant treatment. Written, informed consent was obtained before procuring the tissue for research purposes. Permission to use samples and data was given by the Cancer Biobank Access Committee (License number 12121) in accordance with NHS Research Ethics Committee conditions.

\section{Tissue Microarrays (TMAs) and Immunohistochemistry (IHC)}

Tissue samples were uniformly fixed in $10 \%$ formalin within $30 \mathrm{~min}$ of surgery. Representative areas were marked on $\mathrm{H}$ \& $\mathrm{E}$ sections for TMA construction. TMAs were made in triplicate using a manual arrayer (Beecher Instruments, Sun Prairie, WI, USA) with $0.6 \mathrm{~mm}$ stylet. Each TMA consisted of 85-115 tissue cores, with 5 cores of control tissue samples placed strategically within the block to enable orientation. Sections were cut at $3 \mu \mathrm{m}$ and floated onto polyanionic slides before being dried at $37{ }^{\circ} \mathrm{C}$ overnight followed by incubation for $2 \mathrm{~h}$ at $56{ }^{\circ} \mathrm{C}$. The TMA sections were obtained during the study and freshly stained, as per the recommendations. They were then incubated with the antibodies after establishing appropriate IHC protocols. A two-step, compact, polymer chain, biotin-free IHC protocol on the BOND-MAX ${ }^{\mathrm{TM}}$ (Leica Biosystems, UK) staining system was used with a primary antibody incubation time of $30 \mathrm{~min}$. Antigen retrieval was performed using BOND-MAX Epitope Retrieval solution 1 (Leica Biosystems, UK). The chromogen used was 3,3'-diaminobenzidine (DAB). ER and HER2 status were obtained from patient records. The antibodies are listed in Table 1.

\section{Scoring the immunohistochemical expression of proliferation markers: conventional and digital imaging}

For each of the three markers, a score was determined by assessment of the percentage of invasive carcinoma cells with positively staining nuclei. At least 50 tumour cells per TMA core were considered necessary to ascertain a representative score. Any cores that were folded, absent, or contained an inadequate number of tumour cells were not scored. Conventional scoring was conducted with an Olympus BX50 microscope (Olympus Optical Co., Ltd., Tokyo, Japan) by the first author (SJ) after a period of training and joint scoring.

Slides were subsequently scanned using a Nanozoomer (Hammamatsu, UK), transferred to the digital slide server and accessed online via the Slidepath system (Leica Biosystems, UK). Digital microscopic scoring was performed with the OpTMA scoring software platform (Leica Biosystems, UK) and the percentage of positive nuclei was again assessed similarly to the light microscopic slides. Scoring using each of the two methods was performed independently by the same reader (SJ), one method at a time, and blinded to the results of assessment by the other method. Approximately $10 \%$ of the scores were assessed by more than one author (SJ, JB, PG) and there was in general good agreement among the authors. Since the TMAs were assessed in triplicate, both the maximum (from the 3 cores) and the average of the 3 scores were recorded for final analysis.

\section{Statistical methods}

Where tumours were categorised into two continuous groups, the significance of associations of each of the immunohistochemical scores was assessed with a Mann Whitney test. For clinico-pathological features that grouped tumours into three or more continuous, unpaired categories, a Kruskal-Wallis test was used to assess association. To analyse associations between two continuous variables, Spearman's rank correlation was applied.

Table 1 Antibody panel used for immunohistochemistry

\begin{tabular}{|c|c|c|c|c|c|}
\hline Antigen & Clone & Dilution & Source & System & Scoring method \\
\hline Ki67 & MIB1 & 1 in 75 & Leica & Leica, BOND-Max & As described \\
\hline MCM2 & CRCT2.1 & 1 in 100 & Leica & Leica, BOND-Max & As described \\
\hline Geminin & EM6 & 1 in 30 & Leica & Leica, BOND-Max & As described \\
\hline $\mathrm{ER}$ & SP6 & 1 in 100 & Invitrogen & Leica, BOND-Max & $>2$ Allred \\
\hline HER2 & \multicolumn{2}{|c|}{ Ready to use kit } & Leica & Leica, BOND-Max & $3+$ \\
\hline
\end{tabular}


Wilcoxon signed rank test and Friedman's test were used to evaluate continuous, paired variables of 2 and 3 groups, respectively. All the above statistics were performed using GraphPad PRISM Version 6.0c (GraphPad Software, Inc, CA, USA).

In order to establish a cut-off between high and low expression that enabled the most accurate prediction of breast cancer-specific survival (BCSS) for each of the markers, time-dependent Receiver Operating Characteristic (ROC) curves were created from the censored survival data using the Kaplan-Meier method with the $R$ package survivalRO [24]. The sensitivity and specificity for predicting 15-year BCSS were calculated for various cut-off values using a statistically-determined baseline marker value as reference [25]. The value that yielded the highest balanced accuracy, defined as (sensitivity + specificity)/2, was selected as the optimal cut-off value.

Using the defined cut-off values to categorise cases into high-expressing and low-expressing tumours, Kaplan-Meier survival curves were constructed and compared using the log-rank test for each marker. BCSS was defined as the interval from the date of histological diagnosis to the date of death due to breast cancer up until 15 years. All other causes of death, including those cases where the cause was unknown or was ambiguous, were censored at the last follow-up.

Multivariable analysis was conducted using Cox's regression model with backward stepwise model selection of predictors using the Akaike Information Criterion [26]. The initial set of predictors for the multivariable model included histological grade $(1,2$ or 3 ), age ( $>50$ years or $<50$ years), lymph node status (positive or negative), clinical tumour size $(<2 \mathrm{~cm}, 2-5 \mathrm{~cm}$ or $>5 \mathrm{~cm})$, ER status (Allred $>2$ as positive) and HER2 status (positive if scored $3+$ on IHC or FISH positive). Multivariable analysis was then conducted as before. Subgroup univariable and multivariable survival analyses on ER-positive cases were conducted similarly. All survival analysis was performed in the statistical language $\mathrm{R}$ and is provided as a Sweave document in Supplementary Methods (Additional file 3). In all statistical tests, $P<0.05$ was considered significant.

\section{Results}

\section{Patient and tumour characteristics}

Patient and tumour characteristics are shown in Table 2. In this series of 309 cases, $70.1 \%$ of patients were over 50 years of age, $53.8 \%$ had lymph node-negative disease, $75.6 \%$ were ER-positive and $16.8 \%$ were HER2-positive (although HER2 status was known for only $50 \%$ of patients in this historical cohort). $43.4 \%$ were of histological grade 2 and $55.4 \%$ were between 2 and $5 \mathrm{~cm}$ in size. The median follow-up period was 13 years ( 1 to 17.2 years). The median overall survival was 13.48 years (0.3 to 18.1 years). There were 160 patients who died $(51.8 \%)$ at the end of the follow up period, only 83 of whom were known to have died of breast cancer.

\section{Correlation between proliferation markers and methodology}

To explore the information provided by the scores for each marker, we first compared them across the cohort. We found that a greater proportion of tumour cells showed expression of MCM2 than Ki67 and Geminin, with the latter having the lowest frequency of expression $(P<0.001$; Wilcoxon signed rank test). The median light microscopic scores of Ki67, MCM2 and Geminin when using the maximum score from the 3 TMA cores, were $10 \%, 30$ and $5 \%$, respectively. With the mean light microscopic score from the 3 cores, the median values of Ki67, MCM2 and Geminin expression were $7.7 \%, 24$ and $3 \%$, respectively. With the digital scoring technique, the medians of the maximum scores from the 3 TMA cores were $7 \%, 37$ and $2 \%$ whereas the medians of the average 3 scores were $4.5 \%, 27$ and $2 \%$ for Ki67, MCM2 and Geminin, respectively (Table 3). Representative cores with staining for Ki67, MCM2 and Geminin are shown in Fig. 1a-b, e-f and $\mathrm{i}-\mathrm{j}$, respectively. Frequency distribution curves for the average Ki67, MCM2 and Geminin scores are shown in Fig. 1c, g and k, respectively.

In order to assess inter-core variability within a sample, we compared the expression of Ki67 (110 cases), MCM2 (116 cases) and Geminin (105 cases) across those samples for which all 3 cores were available and found no significant difference for Ki67 or MCM2, $(P=0.411$ for Ki67, $P=0.322$ for MCM2; Friedman's test) indicating that Ki67 and MCM2 expression was consistent across the 3 cores. In contrast, the intercore variability for Geminin was significantly higher $(P<0.006$; Friedman's test). Of note, the average of 2 cores provided comparable results to the average values of 3 cores (Ki67: $r_{s}=0.96, P<0.0001 ;$ MCM2: $\mathrm{r}_{\mathrm{s}}=0.95, \quad P<0.0001 ;$ Geminin: $\left.\mathrm{r}_{\mathrm{s}}=0.95, \quad P<0.0001\right)$ suggesting that one may evaluate 2 or 3 cores for such IHC markers. We also observed that the loss of data due to core loss or absence of sufficient tumour, decreased from $37-40 \%$ to 22 and $16 \%$, if 1,2 or 3 cores were considered respectively for all 3 proliferation markers. The average of the values obtained from 3 cores strongly correlated with the maximum of the 3 (Ki67: $\mathrm{r}_{\mathrm{s}}=0.97, P<0001 ; \mathrm{MCM} 2: \mathrm{r}_{\mathrm{s}}=0.98, P<0.0001 ;$ Geminin: $\left.r_{s}=0.98, P<0.0001\right)$. Since there was little difference between the average and maximum value obtained from 3 cores; we proceeded with the average value for further analysis. 
Table 2 Patient and tumour characteristics of 309 cases of early breast cancer

\begin{tabular}{|c|c|}
\hline Clinico-pathological feature & $\begin{array}{l}\text { Distribution (percentage of cases } \\
\text { with data) }\end{array}$ \\
\hline \multicolumn{2}{|l|}{ Age, years } \\
\hline Median & 58 \\
\hline Range & $28-85$ \\
\hline$<50$ & $92(29.9 \%)$ \\
\hline$>50$ & $216(70.1 \%)$ \\
\hline \multicolumn{2}{|l|}{ Tumour size } \\
\hline$<2 \mathrm{~cm}$ & $114(41.3 \%)$ \\
\hline $2-5 \mathrm{~cm}$ & $153(55.4 \%)$ \\
\hline$>5 \mathrm{~cm}$ & $9(3.3 \%)$ \\
\hline Not known & 33 \\
\hline \multicolumn{2}{|l|}{ LN status } \\
\hline Positive & $132(46.2 \%)$ \\
\hline Negative & $154(53.8 \%)$ \\
\hline Not known & 23 \\
\hline \multicolumn{2}{|l|}{ Histological Grade } \\
\hline 1 & $56(20.1 \%)$ \\
\hline 2 & $121(43.4 \%)$ \\
\hline 3 & $102(36.6 \%)$ \\
\hline Not known & 30 \\
\hline \multicolumn{2}{|l|}{ ER (Estrogen Receptor) status } \\
\hline Positive & $226(75.6 \%)$ \\
\hline Negative & $73(24.4 \%)$ \\
\hline Not known & 10 \\
\hline \multicolumn{2}{|l|}{ HER2 status (IHC 3+ or FISH + ve) } \\
\hline Positive & $26(16.8 \%)$ \\
\hline Negative & $129(83.2 \%)$ \\
\hline Not known & 154 \\
\hline \multicolumn{2}{|l|}{$\begin{array}{l}\text { Recurrence (Local, regional, distant } \\
\text { or death when death was known to } \\
\text { be caused by breast cancer) }\end{array}$} \\
\hline Total & 111/309 (35.9\%) \\
\hline Median time to recurrence (years) & 3.14 \\
\hline Range (years) & $0.05-19.05$ \\
\hline \multicolumn{2}{|l|}{ Mortality } \\
\hline Total deaths with known cause & 148 \\
\hline Deaths due to breast cancer & $83(56 \%)$ \\
\hline $\begin{array}{l}\text { Deaths with breast cancer present } \\
\text { at death }\end{array}$ & $57(38.5 \%)$ \\
\hline $\begin{array}{l}\text { Deaths due to causes other than } \\
\text { breast cancer }\end{array}$ & $8(5.4 \%)$ \\
\hline Not known & 12 \\
\hline \multicolumn{2}{|l|}{ Overall survival (years) } \\
\hline Median & 13.48 \\
\hline Range & $0.33-18.11$ \\
\hline
\end{tabular}

Table 2 Patient and tumour characteristics of 309 cases of early breast cancer (Continued)

\begin{tabular}{ll}
\hline Follow-up (years) & \\
Median & 13 \\
Range & $1-17.2$
\end{tabular}

\section{Comparison between conventional light microscopic and} digital image assessment

We next asked whether there was any appreciable difference between the results obtained from scoring the section using the traditional light microscope as opposed to assessment of the scanned digital image. A significant correlation between the scores of the two techniques was observed for each marker (Ki67: $\mathrm{r}_{\mathrm{s}}=0.87, P<0.001$, Fig. 1d; MCM2: $\mathrm{r}_{\mathrm{s}}=0.94, P<0.001$ Fig. $1 \mathrm{~h}$; and Geminin: $\mathrm{r}_{\mathrm{s}}=0.86, P<0.001$, Fig. 1l; Spearman's rank correlation), with the scores for MCM2 exhibiting the highest concordance.

\section{Association with clinico-pathological features and BCSS}

We investigated whether the immunohistochemical expression of Ki67, MCM2 and Geminin was significantly associated with clinico-pathological features. These analyses were performed using the median value of both the maximum as well as the average values of three TMA cores scores and no significant difference between these two approaches was observed. Whilst tumour size, lymph node status and HER2 status were not associated with any of the three proliferation markers, higher histological grade and ER-negative tumours had higher expressions of all 3 markers, $P<0.001$ for all, Mann Whitney test (Table 4).

Next we investigated if any of the three markers of proliferation possessed prognostic value in our cohort by first using time-dependent ROC curves to calculate cutoffs that yielded the highest balanced accuracy for 15year BCSS. These cut-offs were $8 \%, 12$ and $2.33 \%$ for Ki67, MCM2 and Geminin, respectively (ROC curves for cut-off calculation are shown in Fig. 2b, $d$ and $f$ ). In a univariable Cox regression analysis, high expression of all 3 markers of proliferation was significantly associated with 15 year BCSS using optimal cut-off values for Ki67 $\{P=0.0142, \mathrm{HR}=0.55$ (0.34-0.89); log-rank test showing $95 \%$ confidence intervals $\}$ (Fig. 2a); for MCM2 $\{P=0.0005, \quad H R=0.27 \quad(0.12-0.59) ;$ log-rank test showing $95 \%$ confidence intervals\} (Fig. 2c); and for Geminin $\{P=0.0072, \mathrm{HR}=0.51 \quad(0.31-0.84)$; logrank test showing $95 \%$ confidence intervals\} (Fig. 2e).

To offset some of the heterogeneity that arises from the inclusion of ER/PR negative cases in a consecutive series of patients, we next used the same expression cutoffs and looked within the ER-positive subgroup. We recapitulated the results seen in the wider cohort with 
Table 3 Immunohistochemical expression of Ki67, MCM2 and Geminin in 309 cases of early breast cancer as assessed by light microscope and digital imaging and the correlation between the two methods of scoring

\begin{tabular}{|c|c|c|c|c|c|c|c|c|c|c|}
\hline \multirow[t]{2}{*}{ Marker } & \multirow[t]{2}{*}{ Score } & \multicolumn{4}{|c|}{ Conventional method of scoring } & \multicolumn{4}{|c|}{ Digital method of scoring } & \multirow{2}{*}{$\begin{array}{l}\text { Correlation } \\
\text { Spearman's co-efficien }\end{array}$} \\
\hline & & Available values & Max & Min & Median & Available values & Max & Min & Median & \\
\hline \multirow[t]{6}{*}{ Ki67 } & Maximum of the 3 cores & 258 & 95 & 0 & 10 & 175 & 97 & 0 & 7 & $n=174^{\mathrm{a}}$ \\
\hline & & & & & & & & & & $0.90(0.86-0.92)$ \\
\hline & & & & & & & & & & $p<0.001$ \\
\hline & Average of the 3 cores & 258 & 90 & 0 & 7.7 & 175 & 85.33 & 0 & 4.5 & $n=174^{\mathrm{a}}$ \\
\hline & & & & & & & & & & $0.91(0.88-0.93)$ \\
\hline & & & & & & & & & & $p<0.001$ \\
\hline \multirow[t]{6}{*}{ MCM2 } & Maximum of the 3 cores & 260 & 100 & 0 & 30 & 167 & 100 & 0 & 37 & $n=167^{\mathrm{a}}$ \\
\hline & & & & & & & & & & $0.92(0.90-0.94)$ \\
\hline & & & & & & & & & & $p<0.001$ \\
\hline & Average of the 3 cores & 260 & 100 & 0 & 24 & 167 & 98.5 & 0 & 27 & $n=167^{\mathrm{a}}$ \\
\hline & & & & & & & & & & $0.94(0.91-0.95)$ \\
\hline & & & & & & & & & & $p<0.001$ \\
\hline \multirow[t]{6}{*}{ Geminin } & Maximum of the 3 cores & 258 & 40 & 0 & 5 & 270 & 62 & 0 & 2 & $n=257$ \\
\hline & & & & & & & & & & $0.88(0.85-0.91)$ \\
\hline & & & & & & & & & & $p<0.001$ \\
\hline & Average of the 3 cores & 258 & 28.3 & 0 & 3 & 270 & 37 & 0 & 2 & $n=257$ \\
\hline & & & & & & & & & & $0.90(0.87-0.92)$ \\
\hline & & & & & & & & & & $p<0.001$ \\
\hline
\end{tabular}

${ }^{a}$ The number of cores available for digital scoring was not the same as the number available for scoring conventionally. Hence, only those scored by both techniques were compared with each other

Ki67 $\{P=0.049, \quad$ HR $=0.53(0.28-1.01) ;$ log-rank test showing $95 \%$ confidence intervals\} (Additional file 1A) having the weakest prognostic value, MCM2 the strongest $\{P=0.0148, H R=0.35 \quad(0.15-0.85) ;$ log-rank test showing $95 \%$ confidence intervals $\}$ (Additional file $1 \mathrm{~B}$ ), followed by Geminin $\{P=0.0254, \mathrm{HR}=0.47(0.24-0.93)$; log-rank test showing $95 \%$ confidence intervals $\}$ (Additional file 1C).

To examine the utility of these markers as independent predictors of survival, we also performed multivariable Cox regression analysis with backward stepwise regression, and found only high histological grade $\{P=0.0502, \quad \mathrm{HR}=1.84 \quad(1-3.38)\}$ and lymph node-positive status $\{P=0.0006, \mathrm{HR}=3.9(1.79-8.5)\}$ to be associated with breast cancer-related death within 15 years for all breast cancers irrespective of ER positivity (Table 5). Among ER-positive cases, again only lymph node-positive status $\{P=0.0006, \mathrm{HR}=7.13(2.32-21.89)\}$ remained significantly associated with BCSS following a multivariable analysis (Additional file 2).

\section{Discussion}

We have assessed TMAs of 309 cases of primary invasive breast cancers for the expression of the proliferation markers Ki67, MCM2 and Geminin by IHC using conventional light microscopy and by digital imaging. We observed a significantly positive correlation between the methodologies in assessing all the 3 biomarkers confirming that remote assessment of scanned images is comparable with using light microscopy to score histological glass slides.

The methodological aspects of immunohistochemistry are being increasingly standardised as a consequence of the widespread uptake of automated systems that improve consistency. By extending this approach to include digital imaging and computer-aided systems it may be possible to confer greater objectivity to methods of immunohistochemical scoring [27]. In agreement with our findings, and with a view to implementing these changes, Konsti et al. have developed a virtual microscopy and automated analysis platform, which showed $87 \%$ agreement and a weighted kappa value of 0.57 when compared to visual assessment of Ki67 immunohistochemical expression in breast cancer [28]. Digital microscopy for scoring of scanned images of the TMAs, a high-throughput method, has advantages over the conventional light microscopic method. These include ease of handling compared to manual navigation of a TMA slide: for example, the linking of cores to the predefined TMA 'map' ensures that the core/case are accurately identified and recorded. In addition, the samples can be accessed and evaluated remotely through any computer 

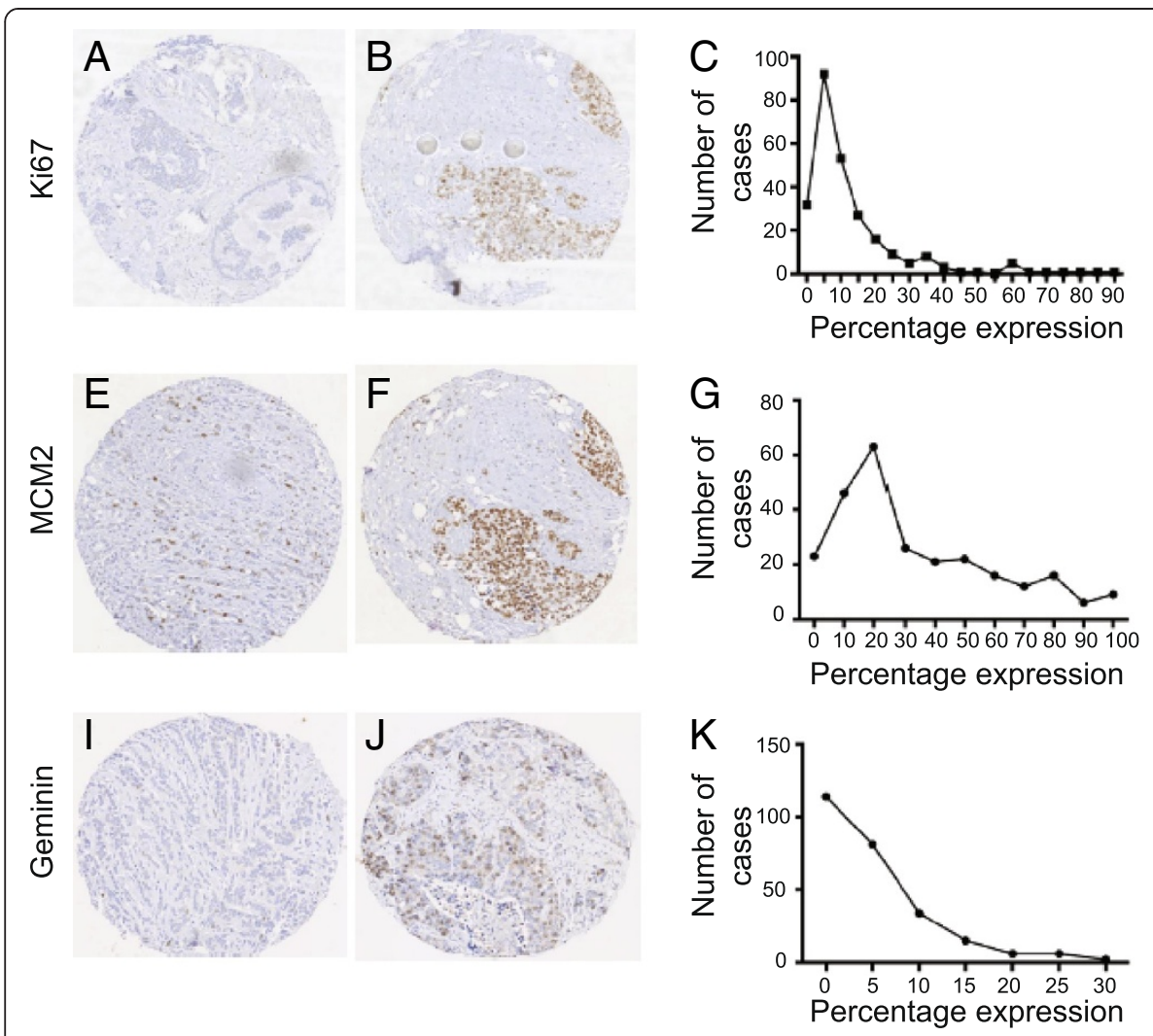
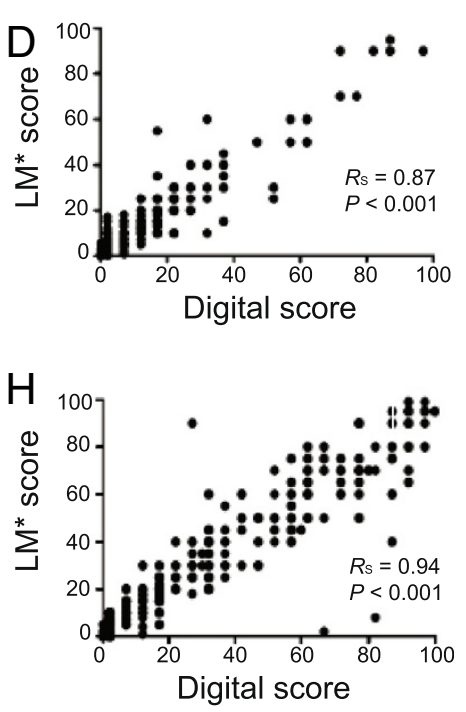

L

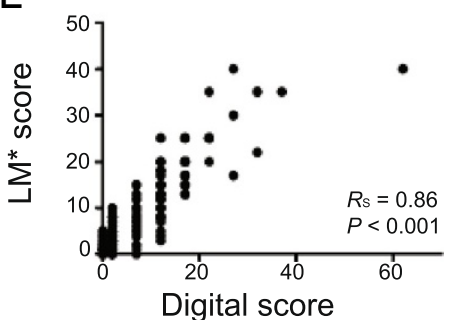

Fig. 1 Expression of proliferation markers in invasive breast cancers. Representative breast cancer cores from a consecutive TMAs showing low and high immunohistochemical staining for 3 proliferation markers Ki67 (a,b), MCM2 (e,f) and Geminin (i,j) (150X magnification). Distribution of IHC determined expression of Ki67 (c), MCM2 (g) and Geminin (k) across 309 primary breast carcinomas. The number of cases is indicated on the $x$-axis, while the percentage scoring for the respective marker is depicted in the $y$-axis. Correlation between light microscopic and digital image guided scores for Ki67 (d), MCM2 (h) and Geminin (I). The Spearman's rank correlation coefficient and p-values are shown

without the need for availability of a light microscope and thus this method provides an opportunity to exchange information between observers, such as the double-reading of slides (particularly valuable for clinical trial material), with ease. Voros et al. used a partially digitised counting method for Ki67, and concluded that such a technique was faster, more convenient and would significantly improve the reproducibility of using Ki67 as a proliferation marker in breast cancer [29].

In this study, we do not report digital image analysis of the cases using computer software but describe the scoring of proliferation marker-stained scanned images by human observers. One of the goals of automated image analysis would be to improve the accuracy and reproducibility in scoring biomarkers such as Ki67, MCM2 and Geminin. Fasanella et al. used computer-assisted image analysis of digitised slides, and found manual and automated methods to be comparable in assessing Ki67 expression in breast cancer $[8,30]$. However, in our opinion, further work is required before automated image analysis can be widely adopted for the determination of proliferation marker frequency in invasive breast cancer patients although our results hint at the potential advantages and non-inferiority to the assessment of digital images over conventional means.

We encountered some recurring questions on the approach to, and methodology of, immunohistochemistry in the TMA setting. TMA technology has been widely used in research and some guidelines for practice are now available [31]. Nonetheless, there are some unresolved issues including the optimum number of cores to be assessed, the extraction of a per-sample score from values obtained from multiple cores (maximum or average), and the calculation of an optimal cut-off for prognostication. For Ki67, we found the average score from two cores to be highly correlated with the average score from three cores. For this marker, using either the average or the maximum from the three cores as the final score, we found little difference in their association to clinico-pathological features, implying that either would be appropriate. Moreover, we observed no significant inter-core variability in Ki67 and MCM2 expression, although Geminin expression differed significantly among the 3 cores. We conclude that for each biomarker study, 
Table 4 Association between the proliferation markers Ki67, MCM2 and Geminin and other prognostic factors in 309 cases of early breast cancer

\begin{tabular}{|c|c|c|c|c|c|c|c|}
\hline Clinico-pathological Feature & Categories & Ki67 median & $p$-value & MCM2 median & $p$-value & Geminin Median & $\overline{p \text {-value }}$ \\
\hline \multirow[t]{2}{*}{$\overline{\mathrm{Age}^{\mathrm{a}}}$} & $</=50$ years & 10 & 0.006 & 31.6 & 0.097 & 3.4 & 0.101 \\
\hline & $>50$ years & 7 & & 21.3 & & 3 & \\
\hline \multirow[t]{3}{*}{ Grade $^{b}$} & 1 & 4.5 & $<0.001$ & 16.5 & $<0.001$ & 0.5 & $<0.001$ \\
\hline & 2 & 7 & & 20.7 & & 2.7 & \\
\hline & 3 & 14.7 & & 50 & & 6.3 & \\
\hline \multirow[t]{2}{*}{ ER status $^{a}$} & Positive & 6.7 & $<0.001$ & 19.1 & $<0.001$ & 2.3 & $<0.001$ \\
\hline & Negative & 14.6 & & 50 & & 8.1 & \\
\hline \multirow[t]{2}{*}{ LN status ${ }^{\mathrm{a}}$} & Positive & 7.5 & 0.787 & 24 & 0.734 & 3 & 0.387 \\
\hline & Negative & 8 & & 24.67 & & 3 & \\
\hline \multirow[t]{2}{*}{ HER2 ${ }^{\text {ac }}$ status } & Positive & 9 & 0.299 & 30 & 0.298 & 3 & 0.039 \\
\hline & Negative & 12.7 & & 37 & & 8.3 & \\
\hline \multirow[t]{3}{*}{ Tumour size ${ }^{b}$} & $</=2 \mathrm{~cm}$ & 7.5 & 0.034 & 23.4 & 0.197 & 2.6 & 0.132 \\
\hline & $>2,<5 \mathrm{~cm}$ & 7.1 & & 24.3 & & 3 & \\
\hline & $>/=5 \mathrm{~cm}$ & 14.5 & & 37.5 & & 5.7 & \\
\hline
\end{tabular}

Mann Whitney test used to test the association between 2 continuous, unpaired variables

${ }^{\mathrm{b}}$ Kruskal-Wallis test used to test the association among 3 continuous, unpaired variables

'Only 155 cases with known HER2 status were included to test the association of HER2 status with each of the proliferation markers

similar analyses are required to evaluate the number of cores required for assessment of that specific lesion, and indeed whether that specific marker can be reliably determined from TMAs at all. Biomarkers with low level expression (such as Geminin) may not be appropriate for TMA studies since reproducible scores from small samples are more problematic than for markers expressed at consistently higher levels (such as MCM2). As a general principle, multiple cores need to be assessed in an attempt to simulate the whole slide and all the representative areas. IHC scoring of a single $1 \mathrm{~mm}$ TMA core for ER/PR/ HER2 was found to be sufficient, without significant heterogeneity by Kyndi and colleagues [32]. Similarly, estimation of Ki67 using TMAs has been proven to have good concordance with whole section assessment [33]. In practice, most studies, including ours, indicate that triplicate core assessment using a $0.6 \mathrm{~mm}$ core size is sufficient for the accurate evaluation of Ki67 and also MCM2 in invasive breast cancer tissue [33, 34].

Different methods of calculating cut-off values for survival analysis have been attempted in the literature, including the dataset median or mean, a literatureinformed value, or an even more arbitrary value [8]. The clinical utility of proliferation marker immunohistochemistry has been largely hampered by the lack of consensus with respect to the cut-off used. In a review by Luporsi and colleagues, Ki67 cut points were distributed between 5 and $38 \%$, with most studies using a cut-off between 10 and $20 \%$ [10]. A multivariable analysis by
Tashima et al. to determine the optimal cut-off for Ki67 revealed $20 \%$ to be the optimal value [35]. In this study, we used time-dependent ROC curves to find the cut-off that yielded the highest balanced accuracy for 15-year BCSS in this patient cohort [25]. The cut-offs we found were lower than those reported in much of the literature. This may reflect our own patient cohort. In addition, the optimal values we report are those we have found to be associated with BCSS as opposed to overall survival (OS) or disease-free survival (DFS), both of which are vulnerable to confounding factors and which are the outcomes reported in other series [36].

As expected, we found ER-negative and high grade tumours to have significantly higher proliferation indices for all 3 markers [37]. Ki67 expression was also significantly associated with tumour size and patient age although none of the three proliferation markers were associated with lymph node or HER2 status (for the number of cases for whom HER2 status was available). These findings are consistent with those from most studies of proliferation markers in breast and other cancers [9].

The proliferation status of a tumour gives an estimate of the rate at which tumour cells enter the cell cycle, which reflects the rate of tumour growth. Ki67 is expressed from late G1 to M phase, MCM2 in all phases and Geminin in the S-G2-M phases of the cell cycle (Fig. 3). This theoretically makes MCM2 a much more sensitive marker of proliferation, since it detects cells that are "licensed to proliferate" and capable of initiating DNA replication [18]. In contrast, Geminin is a more 

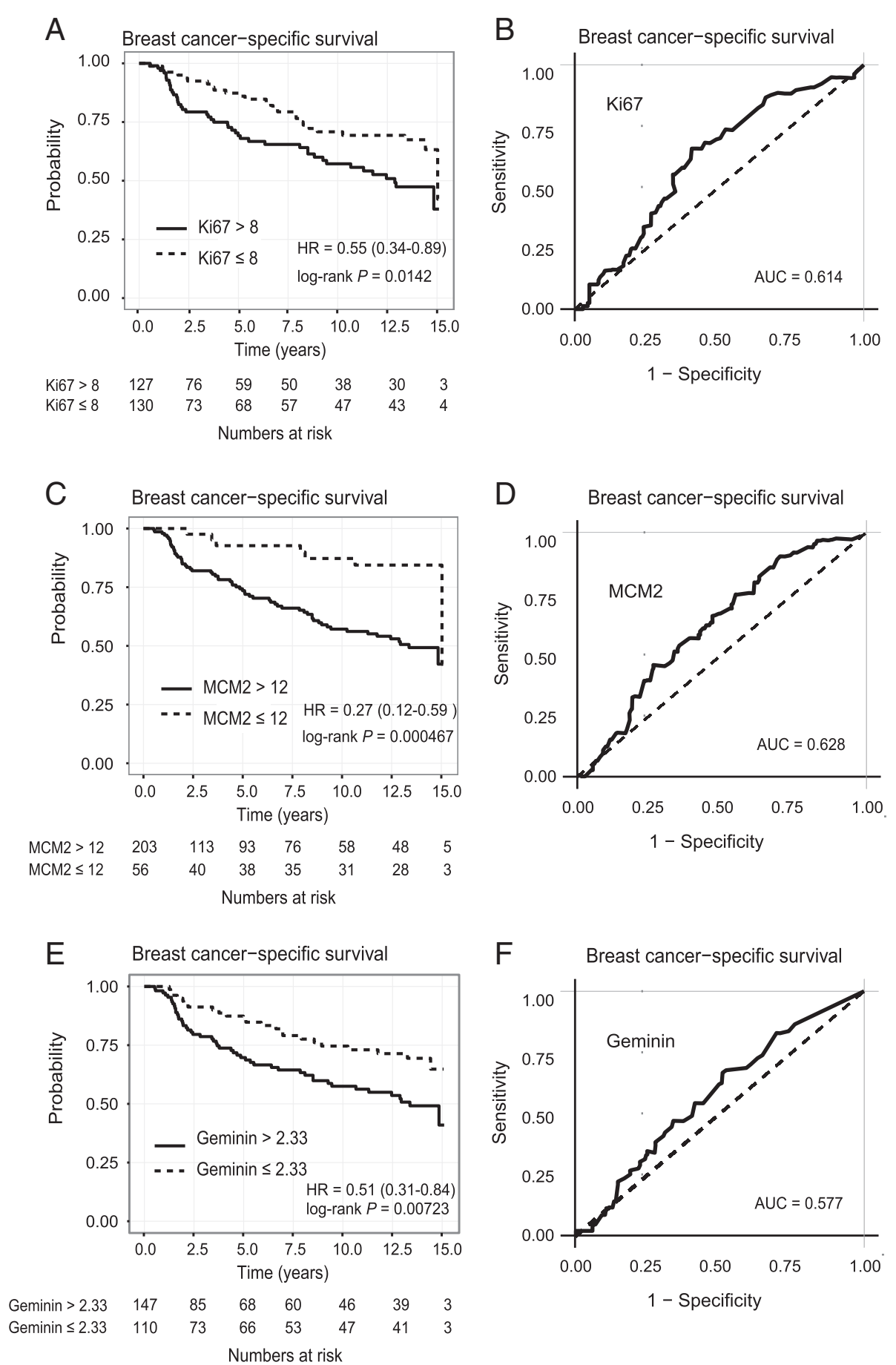

Fig. 2 Univariable breast cancer-specific analyses among 309 invasive breast carcinomas. Kaplan Meier curves showing breast cancer-specific survival (BCSS) in relation to high (solid line) and low (dotted line) expression of Ki67 (a), MCM2 (c) and Geminin (e). The cut-offs of percentage expression were 8, 12 and 2.33 for Ki67, MCM2 and Geminin, respectively. Log rank p-values are stated. The number of patients at risk for every 2.5 years is given for each subgroup. Using time-dependent Receiver Operating Characteristic (ROC) curves for 15-year BCSS, optimal cut-offs were calculated for Ki67 (b), MCM2 (d) and Geminin (f)

specific marker of proliferation, as it only detects cells that are "committed to proliferate" [38]. MCM2 has a significantly higher frequency of expression in breast cancer nuclei than Ki67 and Geminin. Of note, we found
MCM2 to be a more robust and sensitive prognostic marker than Ki67 and Geminin in a univariable survival model, which could be a consequence of these markers being differentially expressed during the cell cycle. In 
Table 5 Univariable and multivariable analyses of prognostic factors for 15-year breast cancer specific survival in 309 cases of early, invasive breast cancer

\begin{tabular}{|c|c|c|c|c|c|c|}
\hline \multirow[t]{2}{*}{ Prognostic factor } & \multicolumn{3}{|c|}{ Univariable Cox regression analysis } & \multicolumn{3}{|c|}{ Multivariable Cox regression analysis } \\
\hline & $\mathrm{HR}$ & $95 \% \mathrm{Cl}$ & $p$-value & $\mathrm{HR}$ & $95 \% \mathrm{Cl}$ & $p$-value \\
\hline Ki67: low & 0.55 & $0.34-0.89$ & 0.014 & 1.38 & $0.56-3.38$ & 0.485 \\
\hline MCM2: low & 0.27 & $0.12-0.59$ & 0.0004 & 0.352 & $0.07-1.85$ & 0.218 \\
\hline Geminin: low & 0.51 & $0.31-0.84$ & 0.007 & 0.824 & $0.34-2.02$ & 0.673 \\
\hline Histological grade: 3 & 1.95 & $1.41-2.69$ & $<0.0001$ & 1.42 & $0.68-2.96$ & 0.346 \\
\hline ER status: positive & 0.32 & $0.2-0.5$ & $<0.0001$ & 0.737 & $0.32-1.72$ & 0.48 \\
\hline HER2 status: positive & 1.65 & $0.84-3.25$ & 0.143 & 0.545 & $0.2-1.5$ & 0.241 \\
\hline LN status: positive & 3.35 & $1.98-5.66$ & $<0.0001$ & 3.44 & $1.54-7.72$ & 0.002 \\
\hline Age $>50$ year & 0.85 & $0.53-1.34$ & 0.477 & 1.36 & $0.63-2.91$ & 0.431 \\
\hline Tumour size: medium & 0.76 & $0.23-2.49$ & 0.891 & 1.38 & $0.16-12$ & 0.772 \\
\hline Tumour size: small & 0.8 & $0.25-2.6$ & 0.891 & 2.84 & $0.32-25.35$ & 0.35 \\
\hline \multicolumn{7}{|c|}{ After backward stepwise regression } \\
\hline Grade: 3 & 1.84 & $1-3.38$ & 0.0502 & & & \\
\hline LN status: positive & 3.9 & $1.79-8.5$ & 0.0006 & & & \\
\hline
\end{tabular}

agreement with our findings, Gonzalez et al. found the MCM2 labelling index to be significantly associated with overall survival and disease free survival in breast cancer and, indeed, that MCM2 was independent of, and superior to, histological grade, Ki67 labelling index and lymph node stage in determining prognosis in a multivariable analysis [20]. Similarly, in a study of oral cavity squamous cell carcinoma, Szelachowska et al. found MCM2 to be prognostically superior to Ki67 in predicting 5year OS [39] whereas the findings of Rodins et al. demonstrated MCM2 to be a better marker of proliferation than Ki67 in normal renal epithelial cells and in different

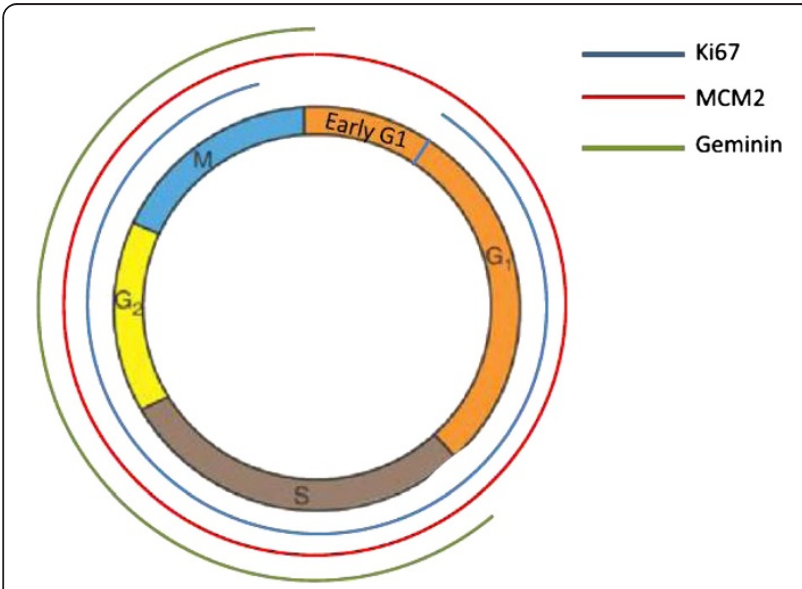

Fig. 3 Differential expressions of the three proliferation markers during the cell cycle. Ki67's expression (shown with a blue line) is detectable from late G1 to M phase. MCM2 (red line) is present in all cell cycle phases. Geminin (green line) is expressed only in the G2-M phase making it a more specific but less sensitive marker of proliferation types of renal tumours, with Ki67 significantly underestimating the number of dividing cells [40]. A number of studies have shown MCM2 expression to be a significant prognostic marker in other tumour types including oesophageal [41] and laryngeal squamous cell carcinoma [42] and oligodendroglioma of the brain [43]. One possible explanation for these observations is the low expression of Ki67 in early G1 phase, which leads to the fraction of cells at this stage of the cell cycle being missed [40]. It thus remains unclear why Ki67 is so utilised in prognostication in invasive breast cancer and other tumours whilst MCM2 is not routinely used.

One potential shortcoming of our study was that all operable, invasive breast cancer cases were included. Subgroup analyses where the assessment of proliferation may be most clinically relevant, for example, of tumours that were lymph node-negative and hormone receptorpositive were not attempted since there were fewer than 100 cases available in our series. One established cut-off for Ki67, as defined by St Gallen's guidelines, is $14 \%$ but this is derived from data on hormone receptor-positive patients. We applied this cut-off in our entire dataset and found the two groups of high versus low expressers had significantly different survivals (data not shown) but our series included both receptor negative and positive disease. In this setting therefore we sought to identify an optimum cut-off for a consecutive cohort of all these operable invasive breast cancers.

Although MCM2 appeared to be more strongly associated with BCSS in a univariable analysis than Ki67, none of the three proliferation biomarkers were independent predictors of survival in a multivariable analysis of the 
whole cohort or of the ER positive sub-population. Lymph node positivity in this series was the most important prognosticator. The greatest utility of assessing proliferation markers may be limited to good prognosis, receptor-positive sub-populations of patients and the clinical utility to select those who are likely to benefit from adjuvant chemotherapy. Of note, different cut-offs may need to be applied to differing sub-types of invasive breast carcinomas to maximise the clinical benefit of determination of proliferation marker expression, which is unlikely to be a 'one size fits all'. However, there were insufficient patients in this good prognosis sub-group for such an approach to be confirmed in the present study.

Inter-observer variation is another critical issue with immunohistochemistry although most studies have shown a good concordance rate. We did not assess inter-observer variation in our study, although there was a general agreement regarding the score amongst the authors. Rather, we focussed on assessing the concordance between scoring by conventional microscopy and by the digital images from the same sections. In the routine setting, inter-observer variation is a potential issue in IHC analyses. Digital imaging with automated scoring may be able to reduce the variability of scoring of IHC. Further research and development into such systems is urgently required.

\section{Conclusions}

We have shown that digital microscopy images can be used as a high-throughput technique for assessing the immunohistochemical expression of proliferation markers in early invasive breast cancer with results that are comparable to those from light microscopy-based scoring. We used MCM2, Ki67 and Geminin, and found MCM2 to be the most sensitive marker of proliferation and prognosis among the three. Despite not finding these three markers to be independently prognostic of BCSS as evinced by our multivariable analysis, digital microscopy-based assessment of these and others may yet find utility in particular subgroups of breast cancer patients, for example in lymph node-negative, hormone receptor-positive patients, which have generally better prognoses. Future studies using immunohistochemistry should be directed towards utility of Ki67 and MCM2 in choosing the appropriate adjuvant therapy in early breast cancer cases.

\section{Additional files}

Additional file 1: Univariable breast cancer-specific analyses among 225 ER-positive cases of invasive breast cancer. Kaplan Meier curves showing breast cancer-specific survival (BCSS) in relation to high (solid line) and low (dotted line) expression of Ki67 (A), MCM2 (B) and Geminin (C). The cut-offs of percentage expression were 8,12 and 2.33 for Ki67, MCM2 and Geminin, respectively. Log rank $p$-values are stated. The number of patients at risk for every 2.5 years is given for each subgroup.
Additional file 2: Univariable and multivariable analyses of prognostic factors for 15-year breast cancer specific survival in 225 cases of ER+ breast cancer.

Additional file 3: Sweave file containing reproducible report forestablishing the immunohistochemical score cut-offs that maximise prognostic accuracy.

\section{Competing interests}

The authors declare that they have no competing interests.

\section{Authors' contributions}

SEP, SJ and PG designed the study. SJ, JPB and PG carried out immunohistochemical staining and scoring of TMAs. SJ and JW conducted the statistical analysis. SJ, JW, AG and SP wrote the manuscript. All authors read, edited and approved the final manuscript.

\section{Authors' information}

Shalaka Joshi and Johnathan Watkins are joint first author.

\section{Acknowledgements}

This research was supported by the Experimental Cancer Medicine Centre at $\mathrm{KCL}$, the National Institute for Health Research (NIHR) Biomedical Research Centre based at Guy's and St Thomas' NHS Foundation Trust and KCL, and Breakthrough Breast Research. Patient tissue samples were provided by King's Heath Partners Cancer Biobank, which is supported by the

Department of Health via the National Institute for Health Research (NIHR) comprehensive Biomedical Research Centre award.

\section{Author details}

'Department of Research Oncology, King's College London, Faculty of Life Science and Medicine, Division of Cancer Studies, Bermondsey Wing, Guy's Hospital, London, UK. 'Breast Cancer Now Unit, King's College London, Faculty of Life Science and Medicine, Division of Cancer Studies, Bermondsey Wing, Guy's Hospital, London, UK. 'King's Health Partners Cancer Biobank, King's College London, Faculty of Life Science and Medicine, Division of Cancer Studies, Bermondsey Wing, Guy's Hospital, London, UK. ${ }^{4}$ Present address: Tata Memorial Centre, Dr E Borges Road, Parel, Mumbai 400012 Maharashtra, India.

Received: 2 January 2015 Accepted: 2 July 2015

Published online: 25 July 2015

\section{References}

1. Sørlie T, Perou CM, Tibshirani R, Aas T, Geisler S, Johnsen H, et al. Gene expression patterns of breast carcinomas distinguish tumor subclasses with clinical implications. Proc Natl Acad Sci U S A. 2001;98(19):10869-74.

2. Ferlay J, Shin HR, Bray F, Forman D, Mathers C, Parkin DM. Estimates of worldwide burden of cancer in 2008: GLOBOCAN 2008. Int J Cancer. 2010;127(12):2893-917

3. Cianfrocca M, Goldstein LJ. Prognostic and predictive factors in early-stage breast cancer. Oncologist. 2004;9(6):606-16.

4. Boyages J, Chua B, Taylor R, Bilous M, Salisbury E, Wilcken N, et al. Use of the St Gallen classification for patients with node-negative breast cancer may lead to overuse of adjuvant chemotherapy. Br J Surg. 2002;89(6):789-96.

5. Hanahan D, Weinberg RA. Hallmarks of cancer: the next generation. Cell. 2011;144(5):646-74.

6. Hall PA, Levison DA. Review: assessment of cell proliferation in histological material. J Clin Pathol. 1990;43(3):184-92

7. Gerdes J, Li L, Schlueter C, Duchrow M, Wohlenberg C, Gerlach C, et al. Immunobiochemical and molecular biologic characterization of the cell proliferation-associated nuclear antigen that is defined by monoclonal antibody Ki-67. Am J Pathol. 1991;138(4):867-73.

8. Yerushalmi R, Woods R, Ravdin PM, Hayes MM, Gelmon KA. Ki67 in breast cancer: prognostic and predictive potential. Lancet Oncol. 2010;11(2):174-83.

9. de Azambuja E, Cardoso F, de Castro G, Colozza M, Mano MS, Durbecq V, et al. Ki-67 as prognostic marker in early breast cancer: a meta-analysis published studies involving 12,155 patients. Br J Cancer. 2007;96(10):1504-13.

10. Luporsi E, André F, Spyratos F, Martin PM, Jacquemier J, Penault-Llorca F, et al. Ki-67: level of evidence and methodological considerations for its role 
in the clinical management of breast cancer: analytical and critical review. Breast Cancer Res Treat. 2012;132(3):895-915.

11. Cheang MC, Chia SK, Voduc D, Gao D, Leung S, Snider J, et al. Ki67 index, HER2 status, and prognosis of patients with luminal B breast cancer. J Natl Cancer Inst. 2009;101(10):736-50.

12. Goldhirsch A, Ingle JN, Gelber RD, Coates AS, Thürlimann B, Senn HJ, et al. Thresholds for therapies: highlights of the St Gallen International Expert Consensus on the primary therapy of early breast cancer 2009. Ann Oncol. 2009;20(8):1319-29.

13. Cuzick J, Dowsett M, Pineda S, Wale C, Salter J, Quinn E, et al. Prognostic value of a combined estrogen receptor, progesterone receptor, Ki-67, and human epidermal growth factor receptor 2 immunohistochemical score and comparison with the Genomic Health recurrence score in early breast cancer. J Clin Oncol. 2011;29(32):4273-8.

14. Dowsett M, Sestak I, Lopez-Knowles E, Sidhu K, Dunbier AK, Cowens JW, et al. Comparison of PAM50 risk of recurrence score with oncotype DX and IHC4 for predicting risk of distant recurrence after endocrine therapy. J Clin Oncol. 2013;31(22):2783-90.

15. Kelly CM, Krishnamurthy S, Bianchini G, Litton JK, Gonzalez-Angulo AM, Hortobagyi GN, et al. Utility of oncotype DX risk estimates in clinically intermediate risk hormone receptor-positive, HER2-normal, grade II, lymph node-negative breast cancers. Cancer. 2010;116(22):5161-7.

16. Mosley JD, Keri RA. Cell cycle correlated genes dictate the prognostic power of breast cancer gene lists. BMC Med Genomics. 2008;1:11.

17. Gwin K, Pinto M, Tavassoli FA. Complementary value of the Ki-67 proliferation index to the oncotype DX recurrence score. Int J Surg Pathol. 2009:17(4):303-10

18. Kearsey SE, Labib K. MCM proteins: evolution, properties, and role in DNA replication. Biochim Biophys Acta. 1998;1398(2):113-36.

19. Yanagi K, Mizuno T, You Z, Hanaoka F. Mouse geminin inhibits not only Cdt1-MCM6 interactions but also a novel intrinsic Cdt1 DNA binding activity. J Biol Chem. 2002;277(43):40871-80.

20. Gonzalez MA, Pinder SE, Callagy G, Vowler SL, Morris LS, Bird K, et al. Minichromosome maintenance protein 2 is a strong independent prognostic marker in breast cancer. J Clin Oncol. 2003;21(23):4306-13.

21. Gonzalez MA, Tachibana KE, Chin SF, Callagy G, Madine MA, Vowler SL, et al. Geminin predicts adverse clinical outcome in breast cancer by reflecting cell-cycle progression. J Pathol. 2004;204(2):121-30.

22. Dudderidge TJ, Stoeber K, Loddo M, Atkinson G, Fanshawe T, Griffiths DF, et al. Mcm2, Geminin, and Kl67 define proliferative state and are prognostic markers in renal cell carcinoma. Clin Cancer Res. 2005;11(7):2510-7.

23. Dowsett M, Nielsen TO, A'Hern R, Bartlett J, Coombes RC, Cuzick J, et al. Assessment of Ki67 in breast cancer: recommendations from the International Ki67 in Breast Cancer working group. J Natl Cancer Inst. 2011;103(22):1656-64.

24. Heagerty PJapbPS-CST-dRcefcsdalhcr-p: Heagerty, P. J. \& packaging by Paramita Saha-Chaudhuri survivalROC: Time-dependent ROC curve estimation from censored survival data. (2013). at < http://cran.r-project.org/ web/packages/survivalROC/. In.

25. Heagerty PJ, Zheng Y. Survival model predictive accuracy and ROC curves. Biometrics. 2005;61(1):92-105

26. Venables WN \& Ripley BD. Modern applied statistics with S. (Springer: New York, 2002).at <http://www.stats.ox.ac.uk/pub/MASS4/. In.

27. Laurinavicius A, Laurinaviciene A, Ostapenko V, Dasevicius D, Jarmalaite $S$, Lazutka J. Immunohistochemistry profiles of breast ductal carcinoma: factor analysis of digital image analysis data. Diagn Pathol. 2012;7:27.

28. Konsti J, Lundin $M$, Joensuu $H$, Lehtimäki $T$, Sihto $H$, Holli $K$, et al. Development and evaluation of a virtual microscopy application for automated assessment of Ki-67 expression in breast cancer. BMC Clin Pathol. 2011:11:3.

29. Vörös A, Csörgő E, Kővári B, Lázár P, Kelemen G, Cserni G. The use of digital images improves reproducibility of the Ki-67 labeling index as a proliferation marker in breast cancer. Pathol Oncol Res; 2013;20(2):391-97.

30. Fasanella S, Leonardi E, Cantaloni C, Eccher C, Bazzanella I, Aldovini D, et al. Proliferative activity in human breast cancer: Ki-67 automated evaluation and the influence of different Ki-67 equivalent antibodies. Diagn Pathol. 2011;6 Suppl 1:S7.

31. Pinder SE, Brown JP, Gillett C, Purdie CA, Speirs V, Thompson AM, et al. The manufacture and assessment of tissue microarrays: suggestions and criteria for analysis, with breast cancer as an example. J Clin Pathol. 2012;66:169-77 doi:10.1136/jclinpath-2012-201091
32. Kyndi M, Sørensen FB, Knudsen $H$, Overgaard M, Nielsen HM, Andersen J, et al. Tissue microarrays compared with whole sections and biochemical analyses. A subgroup analysis of DBCG 82 b\&c. Acta Oncol. 2008:47(4):591-9.

33. Su Y, Shrubsole MJ, Ness RM, Cai Q, Kataoka N, Washington K, et al. Immunohistochemical expressions of Ki-67, cyclin D1, beta-catenin, cyclooxygenase-2, and epidermal growth factor receptor in human colorectal adenoma: a validation study of tissue microarrays. Cancer Epidemiol Biomarkers Prev. 2006;15(9):1719-26.

34. Anagnostou VK, Lowery FJ, Syrigos KN, Cagle PT, Rimm DL. Quantitative evaluation of protein expression as a function of tissue microarray core diameter: is a large $(1.5 \mathrm{~mm})$ core better than a small $(0.6 \mathrm{~mm})$ core? Arch Pathol Lab Med. 2010;134(4):613-9.

35. R Tashima and R Nishimur. Evaluation of an optimal cut-off point for the Ki67 index as a prognostic factorin primary beast cancer. Cancer Res. 2012; Volume 72, Issue 24, Supplement 3. In.

36. Sogaard M, Thomsen RW, Bossen KS, Sorensen HT, Norgaard M. The impact of comorbidity on cancer survival: a review. Clin Epidemiol. 2013;5 Suppl 1:3-29.

37. Jones RL, Salter J, A'Hern R, Nerurkar A, Parton M, Reis-Filho JS, et al. Relationship between oestrogen receptor status and proliferation in predicting response and long-term outcome to neoadjuvant chemotherapy for breast cancer. Breast Cancer Res Treat. 2010;119(2):315-23.

38. Wohlschlegel JA, Dwyer BT, Dhar SK, Cvetic C, Walter JC, Dutta A. Inhibition of eukaryotic DNA replication by geminin binding to $\mathrm{Cdt} 1$. Science. 2000;290(5500):2309-12.

39. Szelachowska J, Dziegiel P, Jelen-Krzeszewska J, Jelen M, Matkowski R, Pomiecko A, et al. Mcm-2 protein expression predicts prognosis better than Ki-67 antigen in oral cavity squamocellular carcinoma. Anticancer Res. 2006;26(3B):2473-8.

40. Rodins K, Cheale M, Coleman N, Fox SB. Minichromosome maintenance protein 2 expression in normal kidney and renal cell carcinomas: relationship to tumor dormancy and potential clinical utility. Clin Cancer Res. 2002;8:1075-81.

41. Going JJ, Keith WN, Neilson L, Stoeber K, Stuart RC, Williams GH. Aberrant expression of minichromosome maintenance proteins 2 and 5 , and Ki-67 in dysplastic squamous oesophageal epithelium and Barrett's mucosa. Gut. 2002;50(3):373-7.

42. Cai K, Luo Y, Li L, Liu Y. [Expression and significance of MCM2, Ki-67 and Rb protein in laryngeal squamous cell carcinomas]. Lin Chung Er Bi Yan Hou Tou Jing Wai Ke Za Zhi. 2012;26:425-8.

43. Wharton SB, Chan KK, Anderson JR, Stoeber K, Williams GH. Replicative $\mathrm{Mcm} 2$ protein as a novel proliferation marker in oligodendrogliomas and its relationship to Ki67 labelling index, histological grade and prognosis. Neuropathol Appl Neurobiol. 2001;27:305-13.

\section{Submit your next manuscript to BioMed Central and take full advantage of:}

- Convenient online submission

- Thorough peer review

- No space constraints or color figure charges

- Immediate publication on acceptance

- Inclusion in PubMed, CAS, Scopus and Google Scholar

- Research which is freely available for redistribution 